Proyectos de la Facultad

\title{
La representación de la ciudad en un sistema de información de viajes
}

Sistema de Información de Viajes / El Departamento de Estudios Tipográficos de la Escuela de Diseño de la Pontificia Universidad Católica de Chile junto a la consultora inglesa Steer Davies Gleave se adjudicaron la propuesta de diagnóstico y actualización del Sistema de Información de Viajes (SIV) del Transantiago, el nuevo plan de transporte público para la ciudad de Santiago, que fue solicitada por el Ministerio de Transporte y Telecomunicaciones en el año 2003. Este estudio, finalizado en agosto de 2006, entregó dos informes con el proceso completo de diseño y un Manual de Normas Gráficas, para regular y especificar la aplicación de los criterios y tecnologías productivas que componen el SIV ${ }^{1}$.

Aquí MANDO yo / El estudio se inició con la etapa de diagnóstico y evaluación del impacto del SIV existente en la ciudad y el comportamiento de sus usuarios. En ese período, el usuario del transporte público de Santiago estaba obligado a recibir (pasivo) u obtener (activo) la información que existía sobre un viaje, en la misma pisadera del bus. Era la opinión de cada chofer, lo que las personas recibían.

Iniciativas para regular el acceso a la información, como INFOBUS ${ }^{2}$, o la aplicación de paradas diferidas en las principales vías de la capital, fueron intermitentes y perdieron fuerza. El usuario, tanto regular como esporádico, no tenía las herramientas que le permitieran organizar su viaje con anterioridad, pues no contaba con una interfaz básica para lograrlo.
Así, la posibilidad de acceder a la malla completa de la ciudad, era una tarea compleja de resolver sin recurrir a voluntades ajenas.

Además de la evaluación del contexto previo, se utilizaron herramientas como grupos focales, encuestas, selección de referentes extranjeros, pruebas de campo y modelos de laboratorio, que sirvieron para establecer parámetros técnicos que le dieran una interfaz a los parámetros culturales definidos en el estudio: conectar lo que genera y utiliza un operador de conocimientos técnicos -como puede ser un ingeniero vial o un ingeniero de sistemas- con lo que el usuario utiliza en su experiencia diaria, para que pueda incorporarlo a su propio lenguaje. En este sentido, el proyecto, básicamente, propulsó el aumento de las instancias de acceso a la información, en relación al momento del viaje y al lugar en la ciudad en que el usuario se encuentre.

Mapeando una CiUdAd que SE mueve / Una de las nuevas piezas que se incorporó al nuevo SIV como una herramienta más en la planificación de viajes, fue el mapa esquemático de Santiago: una representación de los trazados de los servicios del Transantiago en la ciudad. Debido a la gran cantidad de información que el sistema debía dar a conocer, y sobre todo, a las expectativas que un mapa como este podía generar, el equipo planteó la necesidad de conocer cómo las decisiones visuales propias de este proceso ${ }^{3}$ afectarían la percepción de la red de buses y de la ciudad. Para ello, se elabo- raron dos mapas esquemáticos que exploraron las posibilidades de los extremos visuales de representación (fig. 04 y 05) en función de una extensa malla de recorridos, rescatando los elementos positivos en la representación de cada uno de ellos, para poder saber cúales eran las jerarquías en que se accedería a la información y cuáles eran las entidades geográficas (naturales o artificiales) relevantes que se debían incorporar.

Estos dos mapas se desarrollaron con métodos de elaboración similares ${ }^{4}$, sin embargo los aspectos visuales fueron diferentes. El primer mapa tomaba un camino similar a los mapas de metro, cuya principal característica visual es que reduce -y en algunos casos omite- las referencias geográficas, desarrollando trazados geométricos que se estructuran con un mayor orden visual. Esta versión esquemática presentaba sólo la malla de servicios troncales, y se acompañaba de nueve mapas de servicios locales de cada área de la ciudad. El segundo mapa ofrecía una visión geográfica de Santiago, estructurada con un nivel muy bajo de simplificación del trazado vial, acogiendo los accidentes geográficos (cerros, ríos y canales) y los elementos propios de la ciudad (estadios, municipalidades, hospitales o cementerios). Esta versión reunía la malla de servicios troncales junto a la malla de servicios locales.

Estas dos propuestas fueron analizadas en una serie de grupos focales con diferentes usuarios del transporte público, segmenta-
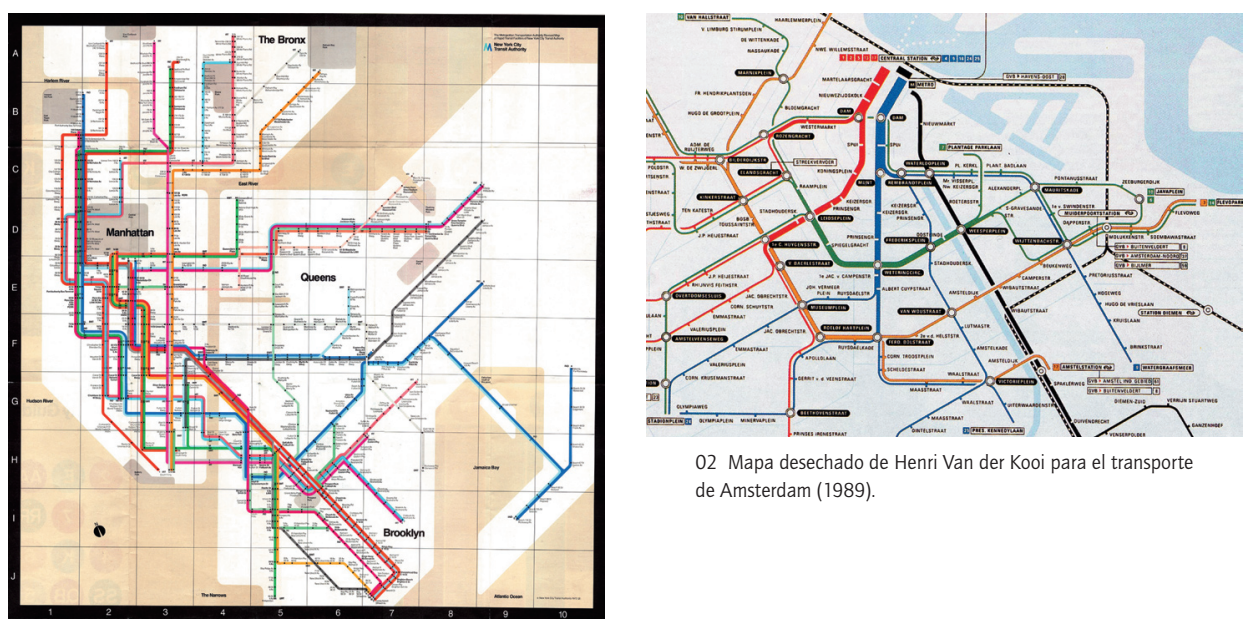

02 Mapa desechado de Henri Van der Kooi para el transporte de Amsterdam (1989).

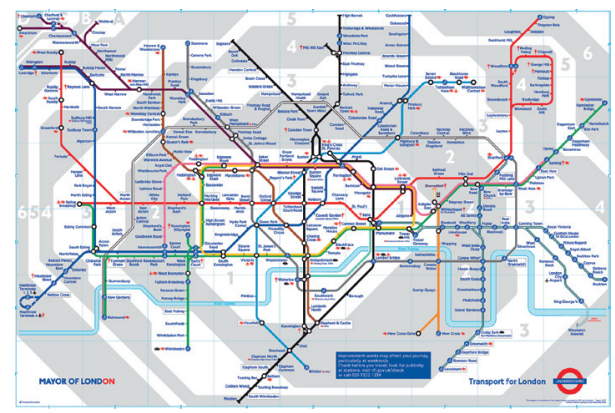

03 Versión de 2007 del mapa de la red de metro de Londres diseñado originalmente por Henry Beck en 1933. Proponía un orden geométrico de la red del metro, utilizando signos abstractos para representar a Londres. 
' Estos estudios, así como el Manual de Normas y los resultados de los grupos focales y encuestas están disponibles para consultas en www. transantiago.cl.

${ }^{2}$ La guía INFOBUS fue un iniciativa lanzada el año 2000 por el Ministerio de Transportes Telecomunicaciones de la época, diseñada por la Máquina del Arte, y tenía por objetivo proveer una efectiva orientación para todos los es y potenciales del transporte público.

3 Para los mapas esquemáticos de Europa Occidental, Alan Morrison define cuatro estilos de mapas. E estilo clásico, el estilo escandinavo, el estilo francés, y el estilo holandés. Estos varían en la cantidad de líneas, color y códigos. Para mas detalles, ver el trabajo de Morrison (1996).

${ }^{4}$ Avelar define cuatro procesos por los que un mapa esquemático puede ser generado: manual, asistido, automático y mecánico. Los mapas desarrollados por el equipo podrían definirse como asistidos, ya que contaban con soft wares gráficos e imágenes proces de la cartografía de Santiago.
${ }^{5}$ El tamaño del mapa fue determinado principalmente, en relación con su portabilidad, su factibilidad de ín y su legibilidad. Para todo e SIV se desarrolló un sistema tipográfico propio, cuyas variantes TS Info y TS Mapa, se relacionan con dos instancias de lectura: distancia de lectura de escala ciudad y escala personal. dos por edad y modo frecuente que utilizan para moverse en la ciudad. Esta experiencia reveló ciertas claves cualitativas para orientar las decisiones, enfrentados a la problemática de representación. Esas claves se agrupaban en tres grandes grupos: primero, la identificación de áreas, comunas y ejes principales. Segundo, el reconocimiento de elementos familiares como referencia de navegación. Tercero, el ruteo, entendido como la interpretación y capacidad de seguimiento.

Estos puntos fueron determinantes para ajustar la relación entre realidad y geometría. Así, en el desarrollo final del mapa, se realizaron mejoras visuales en los trazados de los buses y en los ejes viales más importantes de la ciudad. Además, se confrontaron puntos de vista sobre la relación entre jerarquía y tamaño, lo que se ve reflejado en el aumento del tamaño relativo en zonas de la ciudad de alta concentración de trazados, que permite un mayor entendimiento de zonas complejas ${ }^{5}$ como en los casos del centro histórico y en los centros de San Bernardo, Maipú, Quilicura y Puente Alto, o en la distorsión puntual de áreas como Pudahuel Sur, Huechuraba, Macul o Lo Espejo.

Un TRAJE A LA MEDIDA / Tal como ocurre con el resto de las piezas del sistema, el estudio fue enfático en aclarar que el uso del mapa esquemático requiere de etapas progresivas de aprendizaje y familiarización. Sólo entendiéndolo como un proceso y no como un elemento propio del marketing, cada pieza podrá ocupar el lugar que le fue asignado dentro del SIV, cumpliendo así la etapa inicial proyectada en el estudio. Esta distinción entre información y persuasión es una estrategia que puede llevar tácticas erradas si no se logra poner las cosas en su lugar. En general, en los mapas de buses de superficie, no se observan diferencias muy grandes entre topografía y representación, sin embargo para una malla inicial cercana a los 150 servicios, se necesitaba de un traje a la medida y no de un modelo idealizado de representación esquemática. Puede entenderse que en el caso del mapa de recorridos del metro de Nueva York, de Massimo Vignelli (1972), o en el del mapa de transporte para la ciudad de Amsterdam, de Henri Van der Kooi (1989), la decisión de reemplazarlos por nuevas versiones no tenían justificacio- nes en el ámbito propio del diseño, sino que estaban relacionados con su eficacia cultural como interfaz de un sistema de transporte. El primero por ser una apuesta extrema en el uso de los recursos geométricos para transformar la ciudad y el segundo, porque los habitantes de Amsterdam no podían entenderlo, como lo explica Paul Mijksenaar en su libro Introducción al diseño de información (fig. 01 y 02). Uno podría plantear que es la lejanía con la realidad geográfica lo que los convirtió en mapas desechados. Sin embargo, el mapa del metro de Londres, de Henry C. Beck (1933), que en su concepción visual no se distancia mucho del de Vignelli para Nueva York, luego de más de cincuenta años, es un modelo a seguir, no sólo para muchos sistemas de transporte en el mundo, sino que para el diseño de información en general (fig. 03).

Todos estos ejemplos, que aportan claves en la relación con el ámbito cultural en que un sistema de información de magnitudes urbanas se inserta, nos pueden dar aún más pistas la manera en que los habitantes de esta ciudad se mueven e informan.

\section{Bibliografía}

Avelar, S. y Hurni, L. "On the design of schematic transport maps". Cartographica Vol. $41 \mathrm{~N}^{\circ} 3$ 2006. pp. 217-228. / Avelar, S. Schematic maps on demand: design, modelling and visualization. PhD diss., $\mathrm{N}^{\circ} 14700$, ETH Zúrich, 2002. / Barkowsky, T. y Freksa, C. "Cognitive requirements on making and interpreting maps". En S. Hirtle y A. Frank., eds. Spatial information theory: a theoretical basis for GIS. Springer, Berlín, 1997. pp. 347-361. / Mijksenaar, Paul. Introducción al diseño de la información. Ed. Gustavo Gili, Barcelona, 2001. / Morrison, A. "Public transport maps in western european cities”. Cartographic Journal Vol. 33 N² 1996. pp. 99-110.

Sergio Ramírez Diseñador, Pontificia Universidad Católica de Chile

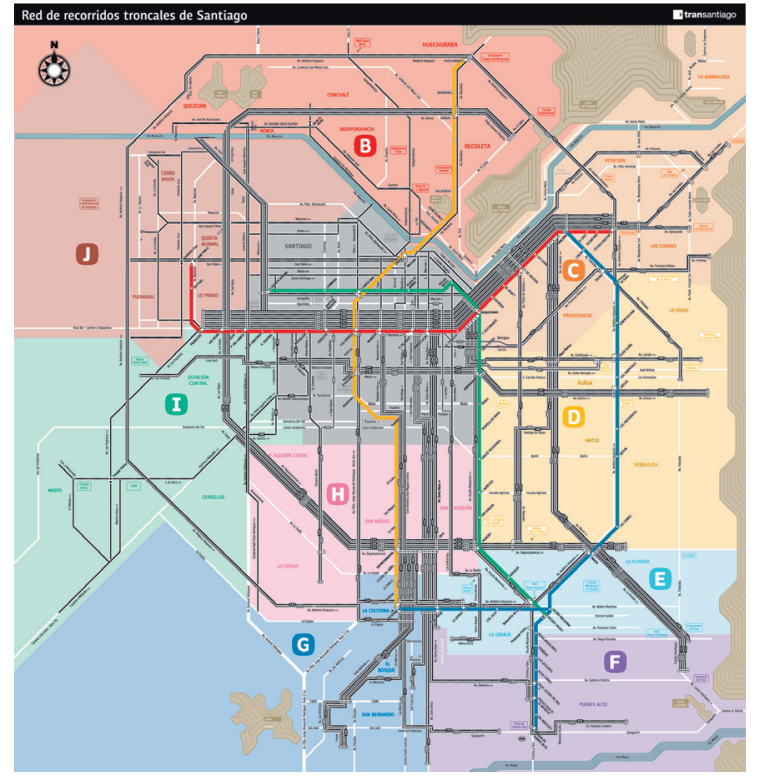

04 Versión esquemática preliminar del mapa para el Transantiago. Alta simplificación de trazos usando ángulos conocidos.

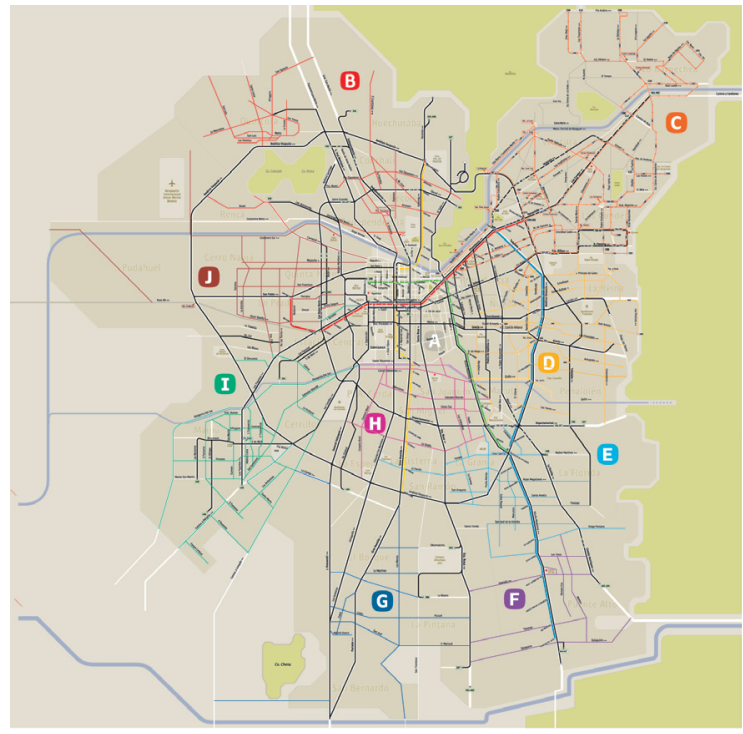

05 Estudio del mapa para el Transantiago. Versión que acoge los accidentes geográficos y las entidades de superficies propias de Santiago.
Dirección del proyecto José Manuel Allard y Rodrigo Ramírez. Diseñadores Francisco Gálvez, Juan Pablo Rioseco, Carola Zurob y Sergio Ramírez.

Ingenieros Steer Davies Gleave. Gloria Hutt, Andrés Roasenda y Rodrigo Correia.

Consultores Julián Naranjo, Héctor Araneda, Gonzalo Cáceres, Lorenzo Shakespear, Francisco Sabatini.

Contraparte técnica Ministerio de Transportes y Telecomunicaciones, Transantiago. 